\title{
Onset and Peak Detection over Pulse Wave Using Supervised SOM Network
}

\author{
A. Orjuela-Cañón, H. Posada-Quintero, D. Delisle-Rodríguez, M. Cuadra-Sanz, R. Fernández de la \\ Vara-Prieto, and A. López-Delis
}

\begin{abstract}
Traditional
methodologies

use electrocardiographic (ECG) signals to develop automatic methods for onset and peak detection on the arterial pulse wave. An alternative method using pattern recognition is implemented to detect onset and peak fiducial points, using Self Organizing Maps (SOM). In the present work SOM neural networks were trained with a dataset of signals with information about localization of onset and peak points. Later on, the trained network was used to make the detection on a validation dataset. This was developed using a shifting temporal windowing, which is presented to the network to decide whether the window corresponds to an onset or peak in the pulse wave. Results of the classification reach $97.93 \%$ over the validation dataset. Sensitivity and positive predictivity measures were used to assess the proposed method, reaching $\mathbf{1 0 0 \%}$ for sensitivity and $\mathbf{9 9 . 8 4 \%}$ for the positive predictivity detecting peaks in the signals. This proposal takes advantages from SOM neural networks for pattern classification and detection. Additionally, ECG signal is not necessary in the presented methodology.
\end{abstract}

Index Terms-Electrocardiography, fiducial points photoplethysmography, self-organizing maps.

\section{INTRODUCTION}

The photoplethysmography (PPG) has been employed as a simple and low-cost optical technique. It is employed for measuring the blood volume changes through the detection of light emission and reception on the skin surface of peripheral body sites (finger, ears, toes and forehead) [1], [2] . Blood volume and perfusion changes, due to the dissemination or absorption of the incident light, provide the dynamical part of the signal.

Applications of PPG signal treatment can be found in commercial medical equipment, where measures of oxygen saturation, blood pressure or heart rate monitoring assess autonomic functions and contribute to peripheral vascular diagnosis. In this way, onset and peak pulse detection on PPG signals is used to obtain relevant information such as pulse transit time (PTT) and pulse wave velocity (PWV), which evaluate vascular effects of aging, hypertension,

Manuscript received November 15, 2012; revised January 28, 2013. This work was supported by the Universidad Antonio Nariño under grant PI/UAN-2012-552Bit and Universidad de Oriente.

A. D. Orjuela-Cañón and H Posada-Quintero are with GIBIO Electronic and Biomedical Faculty, Universidad Antonio Nariño, Bogotá D.C. - Colombia (e-mail: alvorjuela@uan.edu.co, hposada@uan.edu.co).

D. Delisle, R. Fernandez and D. A. Lopez are currently with Medical Biophysics Center in the Universidad de Oriente, Santiago de Cuba, Cuba (e-mail: denis.delisle@cbiomed.cu,_ramon.fernandez@cbiomed.cu, alberto.lopez@cbiomed.cu).

M. Cuadra is with Medical Biophysics Center, Universidad de Oriente, Santiago de Cuba, Cuba (e-mail: manuel.cuadra@cbiomed.cu). stiffness and atherosclerosis [3], [4].

PPG signal typically has small amplitude, its incident and reflected waveform can be affected by conditions as sensor positioning, skin features, breathing, baseline drift, perfusion phenomena, viscoelastic and viscosity property of arteries, arterial stiffness, and reflected waves from peripheral sites. This makes the onset and peak points detection a difficult task [5].

Several methods have been developed for this detection task varying its complexity. These can include adaptive threshold, computer-based filtering, feature extraction, and derivative calculation [6]-[10]. Most of them are assisted by the electrocardiographic (ECG) signal, which provides a cost increment of medical equipment and makes difficult its clinical applications in the Primary Health System.

In [7], morphological similarity of adjacent pulses is used to enhance signal quality and increase the accuracy of the onset pulse detection. A disadvantage of the method is the inclusion of measures from time interval between $\mathrm{R}$ to $\mathrm{R}$ peak of ECG signals. Additionally, principal components analysis (PCA) is applied over adjacent peaks to enhance the onset detection. PCA information, second derivative and tangent intersection in PPG signal show an enhanced accuracy and precision in this approach [8]. Recently, in [9] a new method was presented, based on collected photoplethysmograms. This method does not use ECG signal and works through PPG signal filtering in different ways, but digital filters introduce delays in the temporal signal, which can give wrong information about onset localization in the signal. In [10], a delineator is implemented, using combinatorial amplitude and interval criteria for finding onset and systolic peaks.

Neural networks have been applied for detection of cardiovascular problems, such as QRS detection [11], [12], clustering [13], [14] and applications with PPG signals [15], [16]. These studies show the advantages of this kind of models for pattern recognition. Despite of benefits in different fields, there are not reported works about onset and systolic peak detection on PPG signals, employing these models.

In this paper, it is presented a proposal based on pattern recognition, which uses a self organized map (SOM) to learn the temporal information around onset and systolic peak location on PPG signals during supervised training. Validation is developed using temporal windows, where the network identifies whether an onset or peak is present in the window and its location.

Next section shows materials and methods used in the present study. Details about database and the employed methodology for detection are explained. Section III 
contains results and a brief discussion about these results. Finally, in Section IV some relevant conclusions are extracted about the exploited methodology in this work.

\section{MATERIALS AND METHODS}

Fig. 1 shows the methodology employed for fiducial points detection. The available database was divided to train SOM network and to validate the proposal. The detector works based on this trained SOM, which finds pattern from onset or peaks over a sliding window of signal. Each segment is presented to the detector and the network decides which window corresponds to an onset or a peak from PPG signal. A decision criterion stage locates the point in the signal.

\section{A. Experimental Protocol and Data Collection}

The database is composed by signals from seven volunteers, who participated in the experimental protocol and provide informed consent in accordance with institutional policy. Each subject remains in rest during five minutes in the supine position. Previously, personal and clinical data were collected of each volunteer to the test. Table I shows the age and vital signs of the subjects.

Acquisition was performed by an experimental station, which collected physiological signals of each person. This station simultaneously acquires electrocardiography and photoplethysmographic signals through an ECG channel with bandwidth between 0.15 to $150 \mathrm{~Hz}$ and two PPG channels with bandwidth from 0.5 to $16 \mathrm{~Hz}$. PPG sensors have an infrared diode (wavelength of $780 \mathrm{~nm}$ ) and photodiode for capture by reflection mode the blood volume changes on skin surface. All channels were sampled to 1000 samples/s and the experimental station was approved by ethical committee. Simultaneous ECG and PPG signals were manually marked by trained observers. This task was developed with support of software tools, which provide additional capabilities such as, add, move and remove the fiducial points of ECG and PPG signals. These points correspond to peaks values of QRS complex and, onset and peaks of PPG signal.

Information about marked onset and peak points by expert observers is useful for testing the proposed method.

\section{B. SOM Training Process}

SOM neural networks are capable of arranging the input data into a discretized two-dimensional space known as map, which attempts to preserve the topological properties of the input space. This approximation can be considered as a nonlinear generalization of principal component analysis [17].

In this case, segments of PPG signal are used to extract onset and peak patterns. The dataset is divided into training and validation sets, signals from five subjects compose the training set and last two the validation set. Each signal in the training set is segmented in windows and labeled according to fiducial points located in the middle of the window.

Based on the annotations of the expert observers, a segment with the marked point (onset or peak) is taken. These fiducial points are located in the middle of segment (Fig. 2). Sets of segments or windows compose the training dataset, which is presented as input to the SOM neural network. The window size is found by experimentation, values of 21, 41, 61, 81 and 101 milliseconds were used. Smaller sizes were not considered because are shorter windows to pattern representation and can be confused with noise segments. In PPG signals, distance between onset and peak is less than 100 milliseconds, due to its nature [18], [19]. For this reason, an upper period of time was not considered. Windows with the same size of the onset and peak pattern were extracted to create a third class called noise, these segments were taken from samples before and after of the onset and peak windows. This makes that the noise windows do not belong to any signal segment employed in other classes.

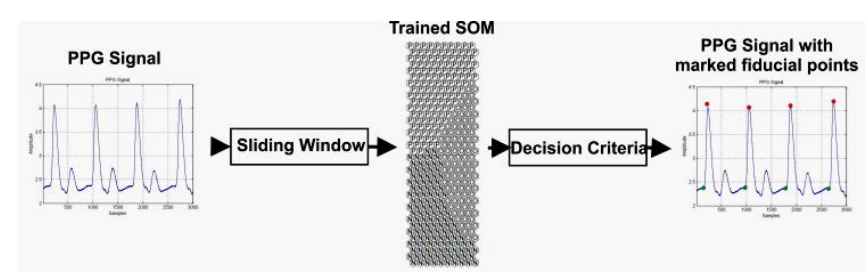

Fig. 1. Detection methodology.

TABLE I: CHARACTERISTICS OF VOLUNTEERS

\begin{tabular}{ccccccc}
\hline \hline Subject & Sex & $\begin{array}{c}\text { Age } \\
(\mathbf{y e a r s})\end{array}$ & $\begin{array}{c}\text { HR } \\
(\mathbf{b p m})\end{array}$ & $\begin{array}{c}\text { SBP } \\
(\mathbf{m m H g})\end{array}$ & $\begin{array}{c}\text { DBP } \\
(\mathbf{m m H g})\end{array}$ & $\begin{array}{c}\text { Breath } \\
\text { /min }\end{array}$ \\
\hline 1 & $\mathrm{M}$ & 19 & 72 & 102 & 68 & 20 \\
\hline 2 & $\mathrm{M}$ & 20 & 60 & 98 & 60 & 16 \\
\hline 3 & $\mathrm{M}$ & 22 & 80 & 125 & 80 & 17 \\
\hline 4 & $\mathrm{M}$ & 20 & 84 & 112 & 72 & 18 \\
\hline 5 & $\mathrm{~F}$ & 18 & 80 & 110 & 64 & 16 \\
\hline 6 & $\mathrm{~F}$ & 18 & 88 & 108 & 58 & 22 \\
\hline 8 & $\mathrm{~F}$ & 18 & 72 & 108 & 68 & 19 \\
\hline \multirow{2}{*}{ Mean \pm SD } & $19.3 \pm 1$ & $76.57 \pm 9$. & $109 \pm 8.5$ & $67.14 \pm 7$. & $18.28 \pm$ \\
& .50 & 36 & 9 & 47 & 2.21 \\
\hline \hline
\end{tabular}

HR: Heart Rate

SBP: Systolic blood pressure

DBP: Diastolic blood pressure

SD: Standard deviation

SOM uses onset, peak and noise classes to do a representation across a nonlinear mapping in an output space with reduced dimensionality. This new space is taken to analyze the original dataset in a graphical way, where different areas of the map preserve characteristics of the classes employed in the training process. This is motivated by the behavior of visual, aural and sensory areas of human cerebral cortex [20].

The learning process consists of three stages: competitive, cooperative and adaptive. In competitive learning, Euclidian distance (weights) from each input to all units or neurons is computed. The unit with more similar weight to the input is defined as the best matching unit (BMU). Then a cooperative process is given around BMU, and units close to it are updated based on a neighborhood function. Finally, adaptive process changes BMU weights according to the input [17]. This is reached through the expression:

$$
w_{i}(t+1)=w_{i}(t)+\eta(t) h_{i j}(t)\left(x(t)-w_{i}(t)\right)
$$

where $w_{i}(t)$ are weights of the map, $\eta(t)$ is a learning coefficient, $h_{i j}(t)$ is a neighborhood function and $x(t)$ is the input vector. 
For training SOM network is necessary to proportionate: number of units, size, type of lattice map and neighborhood function parameters. Number of units and size define the map resolution, type of lattice defines units arrangement from regular or irregular forms, and the base size of the neighborhood function controls cooperative process [17], [20].

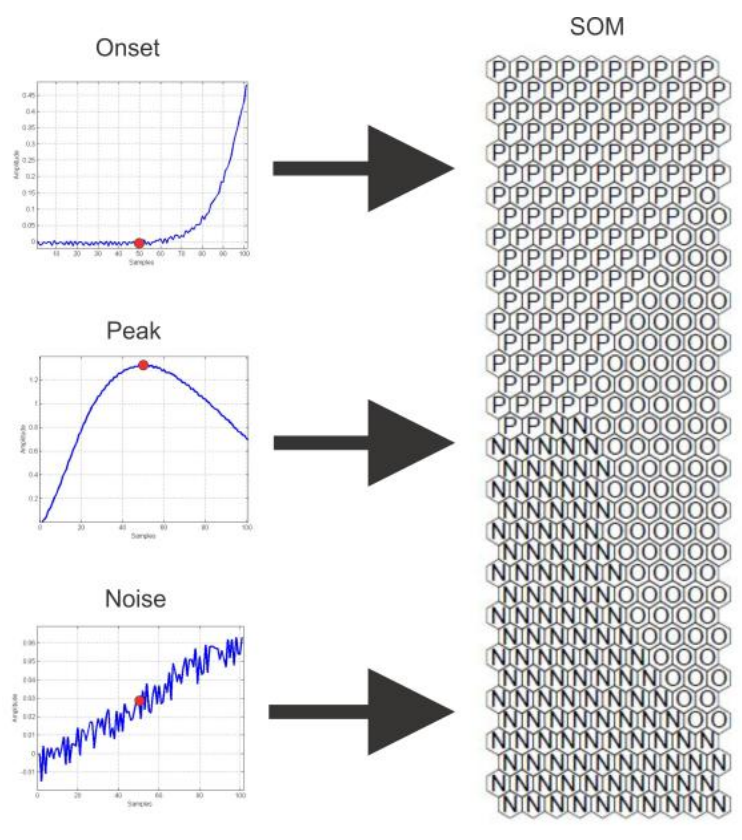

Fig. 2. Input patterns presentation to the SOM neural network.

There are heuristical rules to compute the number of units and the dimension map, one of them is based on principal component analysis (PCA). The ratio of first and second principal components from the training dataset can be an initial value for obtaining the length and width relation of the map [20]. In addition to that, it is attempted what all units have been activated by the data. These rules were followed to determine the number of units and size. Hexagonal topology for lattice was implemented because the distance between adjacent units at the beginning of the training is the same.

Finally, neighborhood function establishes how strong the link between units is. In the present work, it is based on Gaussian distribution, given by:

$$
h_{i j}(t)=\exp \left(-d_{i j}^{2} / 2 \sigma^{2}(t)\right)
$$

where $d_{i j}$ is the Euclidian distance between the $j$ unit and BMU, and $\sigma(t)$ is the basis of the function in the iteration $t$. This parameter changes during the training, beginning with a basis of four units and ending with just a one unit.

The map size and area where the neighborhood function has significant values determine accuracy and generalization of classification.

As the classes of each window are known, the training of the SOM is developed in a supervised way. This assists the detection in a manner that when a new window is presented, the network can classify the signal segment into the predefined classes. It is important that all units of the map have been activated by any input pattern because units without activation can confuse the classification.

\section{Fiducial Points Detection}

SOM trained is used over the validation set and accuracy is measured using fiducial points information as performed in the training set. This provides information about generalization, so that new inputs are presented to map and classification should not have relevant changes.

For onset and peak detection, a sliding windowing in time is developed, where each temporal segment is presented to the trained SOM. From the training information stored by the map, each window is classified as onset, peak or noise segment.

From training, segments with fiducial points as central points were employed (Fig. 2). Adjacent windows to these marked segments also presented activation of the map, yielding false detections. This happens when the window was slid a sample of the real onset or peak windows and the map classifies that segment as onset or peaks. For this reason, it is necessary to implement a decision criterion to reduce this problem.

Adjacent windows of onset or peak segments are classified as onset or peak, too. This happens because the map considers that have the same pattern. Hence, it is necessary to study how to deal with for avoiding activations where the fiducial point is not the centre of window.

It was found that when a segment is presented to the map, output from SOM is the same after sliding the segment a continuous number of samples. Experimentally, time interval for this continuity was measured as $60 \%$ from window size around fiducial point location. In this range, output from map belongs to the same fiducial point. Then, it is necessary to represent all these segments $(60 \%$ around point) as just one fiducial point.

Location of onset or peak point was placed in the last moment of continuity explained before. This information was used as detected point and later on compared with fiducial points marked by expert observers.

Measures of sensitivity (SE), positive predictivity (P) and failed detection rate (FDR) are used to evaluate the method and compare with other approaches. SE indicates the proportion of detected true points in the PPG signal, expression (3) shows its computation. Positive predictivity is based on the percentage of detected true points in relation to all marked points, this can be compute as in (4). Finally, failed detection rate was calculated by (5) and shows how much of the detection is failed.

$$
\text { Sensitivity }=\frac{\text { TruePositive }}{\text { TruePositive }+ \text { FalseNegative }} \times 100
$$

Positive_predictivity $=\frac{\text { TruePositive }}{\text { TruePositive }+ \text { FalsePositive }} \times 100$

FailedDetectionRate $=\frac{\text { FailedDetection }}{\text { NumberofPoints }} \times 100$

\section{RESUlTS AND DisCUSSION}

As mentioned, windows with 21, 41, 61, 81 and $101 \mathrm{~ms}$ 
were used to detect fiducial points. Best results were found with the largest window, where accuracy measured using the validation set reaches $97.93 \%$ of classification. This happens because smaller onset windows are confused with noise segments. Larger segments for pattern representation are easily learned by SOM, making a better classification. Table 2 shows the confusion matrix for results with the largest window. The results are obtained using SOM segments classification from validation dataset. In this case, from 702 onset points, map classifies 673 as onset points and 29 as noise points (total error of $2.06 \%$ ). All peak points have a correct classification.

TABLE II: CONFUSION MATRIX FOR CLASSIFICATION USING 101 MILLISECONDS

\begin{tabular}{lccc}
\multicolumn{4}{c}{ MILLISECONDS } \\
\hline \hline Onset & Peak & Noise \\
Peak & 673 & 0 & 29 \\
\hline \hline
\end{tabular}

Fig. 3 shows the trained map for a $101 \mathrm{~ms}$ window. Areas in the map are labeled with $\mathrm{O}$ for onset, $\mathrm{P}$ for peak and $\mathrm{N}$ for noise in the detection. U-matrix shows the distance between units, dark tones mean short distances and light tones mean large distances. It is possible to observe three areas in the U-matrix, which correspond to each class pattern. Map size was defined as explained before and this depends on the set used in the training, because the ratio between first and second principal components changes, modifying the dimension of the map. In this case a map of $36 \times 10$ units was used to the detection.

Table III and IV show the results of the three described measures for onset and peak detection. Two signals were used for validation of the method. It is possible to appreciate how the modification of the window size improves the sensitivity for onset detection. The best results for onset detection in PPG signal is reached with a window of 101 milliseconds, where this value is $100 \%$ in both signals (Table III).

Positive predictivity in the onset detection case is $83.60 \%$ average. This means that the used method presents a considerable proportion of false positive detections. For peak detection (see Table IV), sensitivity reaches $100 \%$ for windows of 21,61 and $81 \mathrm{~ms}$, which indicates that the window size is not relevant for detection. This can be explained by the peak waveform, which can be represented with a period of time shorter that contain the characteristic curve of this pattern. Positive predictivity for this sensitivity value reaches $84.81 \%$ in mean, when window size corresponds to $61 \mathrm{~ms}$.

It is important to note that the best positive predictivity (99.84\% on average) is given for $101 \mathrm{~ms}$ window in the peak detection case, but sensitivity is not the best, reaching $88.19 \%$ as an average.

The failed detection measure analysis can be extracted by the sensitivity analysis. These results compared with used methods in [7]-[9] are quite close in terms of sensitivity measures. There, sensitivity values reach $99.58 \%$ and $99.17 \%$ when frequency analysis is used. For positive predictivity, the results drop around $10 \%$ compared with cited works [4], [7]-[9]. It is important to say that the present study does not use the ECG signal for detection.

\section{CONCLUSSIONS}

A proposal for onset and peak detection has been presented, based on SOM network trained in a supervised way. It is possible to see that this kind of neural networks learn from a training dataset and generalize this knowledge in a validation set. Patterns from onset and peak segments of the PPG signal are captured by the map and then are used in a temporal analysis of the signal.

A study of the appropriate segment size was implemented. It was observed that a map with a $100 \mathrm{~ms}$ window as input has a better sensitivity for onset points detection.
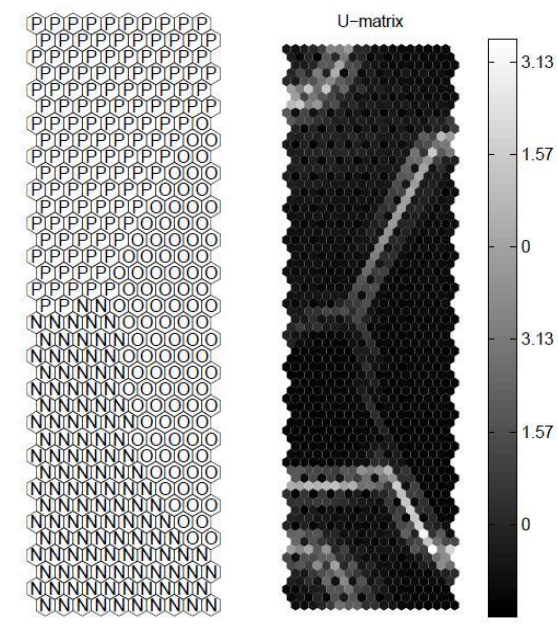

Fig. 3. Trained SOM and U-matrix.

TABLE III: RESULTS FOR ONSET DETECTION

\begin{tabular}{cccc}
\hline \hline Window Size (ms) & SE(\%) & $+\mathbf{+ P}(\%)$ & FDR(\%) \\
\hline 21 & 76.8072 & 18.9169 & 23.1928 \\
21 & 38.9189 & 11.8227 & 61.0811 \\
41 & 95.1807 & 42.7605 & 4.8193 \\
41 & 48.9189 & 23.8158 & 51.0811 \\
61 & 100.0000 & 48.7518 & 0 \\
61 & 97.2973 & 46.9974 & 2.7027 \\
81 & 100.0000 & 85.3470 & 0 \\
81 & 99.7297 & 86.8235 & 0.2703 \\
101 & 100.0000 & 67.4797 & 0 \\
101 & 100.0000 & 99.7305 & 0 \\
\hline \hline
\end{tabular}

TABLE III: RESULTS FOR PEAK DETECTION

\begin{tabular}{cccc}
\hline \hline Window Size $(\mathbf{m s})$ & $\mathbf{S E}(\boldsymbol{\%})$ & $\mathbf{+ P}(\boldsymbol{\%})$ & $\mathbf{F D R}(\boldsymbol{\%})$ \\
\hline 21 & 100.0000 & 52.6149 & 0 \\
21 & 100.0000 & 97.6253 & 0 \\
41 & 85.2410 & 84.9850 & 14.7590 \\
41 & 5.1315 & 5.1213 & 94.8649 \\
61 & 100.0000 & 69.8947 & 0 \\
61 & 100.0000 & 99.7305 & 0 \\
81 & 100.0000 & 62.2889 & 0 \\
81 & 100.0000 & 99.7305 & 0 \\
101 & 99.0964 & 99.6970 & 0.9036 \\
101 & 77.2973 & 100.0000 & 22.7027 \\
\hline \hline
\end{tabular}

For detection of peak fiducial points, a segment with 61 ms obtains the best performance. For onset fiducial points detection, $101 \mathrm{~ms}$ is the period of time of the window appropriated in this system.

The results show that the method reaches good sensitivity values without ECG signal assistance. This is taken as advantage when the resources to develop the detection are restricted.

Additional studies with other datasets can improve the 
method and obtain better results. In this way, comparison with different pattern recognition techniques is one of the future works in this kind of approaches.

\section{ACKNOWLEDGMENT}

This work was supported under grant: PI/UAN-2012-552Bit from Universidad Antonio Nariño, Colombia. Authors want to thank to Universidad de Oriente in Cuba and Dr. Juan Carlos Garcia Naranjo by support this work.

\section{REFERENCES}

[1] J. Allen, "Photoplethysmography and its application in clinical physiological measurement," Physiological Measurement, vol. 28, pp. 1-39, 2007

[2] A. V. Challoner, "Photoelectric plethysmography for estimating cutaneous blood flow Non-Invasive," Physiological Measurements, vol. 1, pp. 125-130, 1979.

[3] T. W. Hansen, J. A. Staessen, C. T. Pedersen, S. Rasmussen, L. Thijs, H. Ibsen, and J. Jeppesen, "Prognostic value of aortic pulse wave velocity as index of arterial stiffness in the general population," Circulation, vol. 113, pp. 664-70, 2006.

[4] N. Bistra and I. Ivo, "An automated algorithm for fast pulse wave detection," Bioautomation, vol. 14, pp. 203-216, 2010.

[5] N. Lyengar, C. K. Peng, R. Morin, A. L. Goldberger, and L. A. Lipsitz, "Age-related alterations in the fractal scaling of cardiac interbeat interval dynamics," American Journal of Physiology (Regulation, Integration and Comparative Physiology), pp. R1078-R1084, 1996.

[6] K. R. G. Egidijus and V. Arunas, "Mathematical methods for determining the foot point of the arterial pulse wave and evaluation of proposed methods," Inform. Technol. And Control, vol. 3, pp. 29-36, 2005.

[7] P. Xu, M. Bergsneider, and X. Hu, "Pulse onset detection using neighbor pulse-based signal enhancement," Medical Engineering and Physics, vol. 31, pp. 337-345, 2009.

[8] S. Hang, L. Chungkeun, and L. Myoungho, "Adaptive threshold method for the peak detection of photoplethysmographic waveform," Computer in Biology and Medicine, vol. 39, pp. 1145-1152, 2009.

[9] C. Liangyou, T. Andrew, and R. Jaques, "Automated beat onset and peak detection algorithm for field-collected photoplethysmograms," IEEE EMBS, pp. 5689-5692, 2009.

[10] B. N. Li, M. C. Dong, and M. I Vai, "On a delineator for arterial blood pressure waveforms," Biomedical Signal Processing and Control, vol. 5, Issue 1, pp. 76-81, January 2010.

[11] U. M. Kacsmar and B. Kordas, "Mining of Electrocardiogram," XXI Autumn Meeting of Polish Information Processing Society, Conference Procedings, pp. 169-175, 2005.

[12] F. Acquaticci, "Detección de Complejos QRS mediante Redes Neuronales," Buenos Aires Argentina, 2007.

[13] M. Lagerholm, C. Peterson, G. Braccini, L. Edenbrandt, and L. Sornmo, "Using Hermite Functions and Self- Organized Maps," IEEE On Transaction on Biomedical Engineering, vol. 47, no. 7, July 2000.

[14] Y. Wenyu, L. Gang, L. Ling, and Y. Qilian, "ECG Analysis based on PCA and SOM," IEEE Int. Conf. Neural Networks 8 Signal Processing, Nanjing, China, December 14-17, 2003

[15] A. Johansson, "Neural network for photoplethysmographic respiratory rate monitoring," Medical And Biological Engineering and Computing, vol. 41, no. 3, pp. 242-248, 2003.

[16] M. Soltane, M. Ismail, and Z. A. A. Rashid, "Artificial neural networks (ANN) approach to PPG signal classification," International Journal of Computing and Information Sciencies, vol. 2, no. 1, April 2004.

[17] S. Haykin, Neural Networks: A Comprehensive Foundation, Third Edition, Prentice Hall, 1998.

[18] F. Camacho, "Statistical analysis of central aortic blood pressure parameters derived from the peripheral pulse" $\mathrm{Ph}$. D dissertation University Of New South Wales, Sydney, Australia, 2005.

[19] R. D. Latham, N. Westerhof, P. Sipkema, B. J. Rubal, P. Reuderink, and J. P. Murgo, "Regional wave travel and reflections along the human aorta: A study with six simultaneous micromanometric pressures," Circulation, vol. 6, pp. 1257-1269, 1985.

[20] T. Kohonen, Self-Organizing Maps, Springer, Third Edition, 2000.

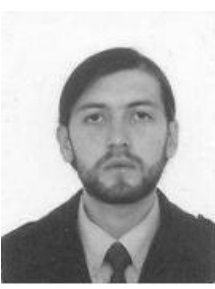

Alvaro D. Orjuela-Cañón received the B.Sc degree in electronic engineering in Bogotá, Colombia in 2006 from Universidad Distrital Francisco José de Caldas. M.Sc degree in electrical engineering from Universidade Federal de Rio de Janeiro in Brazil in 2009. At the same period of time, was with Electrical Energy Research Centre (CEPEL) in Brazil. Currently, he is cursing Ph. D studies in same university. $\mathrm{He}$ is an Assistant professor from Universidad Antonio Nariño in Bogotá, Colombia since 2010. He is with GIBIO research group of the electronic and biomedical engineering faculty. In his topics of interest are signal processing, neural networks, learning machine and diagnosis methods.

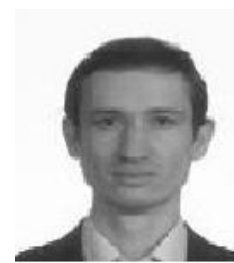

Hugo F. Posada-Quintero received the B.Sc degree in electronic engineering in Bogotá, Colombia from Universidad Distrital Francisco José de Caldas in 2005, the M.Sc degree in electronic and computers engineering from Universidad de los Andes in 2008 Currently, he is pursuing a $\mathrm{PhD}$ at the Worcester Polytechnic Institute, Massachusetts. He has been assistant professor at Universidad Antonio Nariño in Bogotá Colombia since 2008. He is with GIBIO research group of the electronic and biomedical engineering faculty. His topics of interest are mainly biomedical signal processing and biomedical instrumentation.

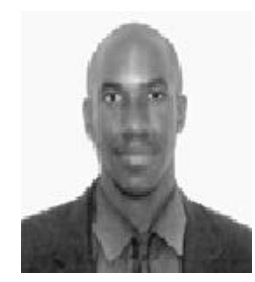

Denis Delisle Rodríguez received the B.Sc. degree in telecommunications and electronic engineering in Santiago de Cuba, Cuba in 2005 from Universidad de Oriente. Currently, he is cursing the M.Sc studies in Biomedical engineering from Universidad de Oriente. Instructor professor from Universidad de Oriente since 2008. He is working in motor neurorehabilitation research group with Medical Biophysics Center from Universidad de Oriente. In his topics of interest are signal processing, neural networks, learning machine and diagnosis methods.

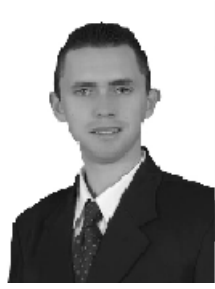

A R. Ramón Fernández de la Vara Prieto received the B.Sc degree in biomedical engineering in Santiago de Cuba, Cuba in 2009 from Universidad de Oriente. Instructor professor from electrical engineering faculty of Universidad de Oriente since 2011. He is researcher at Medical Biophysics Center at the Universidad de Oriente. In his topics of interest are biomedical digital signal processing and biomedical instrumentation.

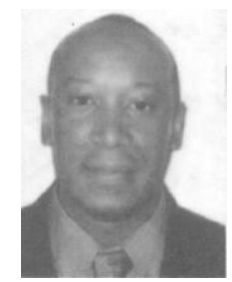

Alberto L. Delis received the B.Sc degree in computer machine engineering in Havana, Cuba in 1992 from Pedagogical Superior Institute José Antonio Hecheverría, M.Sc degree in Automatic Control from University of Oriente in Cuba in 2004 and Ph.D. degree in electrical engineering from the University of Brasilia (UNB), Brasilia, Brazil, in 2010. He is currently a Research Engineer with the Biophysics Medical Center, and is an Instructor Professor in the Electrical Department in the Universidad de Oriente since 2011. His current research interests include biological signal processing, pattern recognition-based control for myoelectric prostheses and exosqueleton, functional electrical stimulation and biomedical instrumentation.

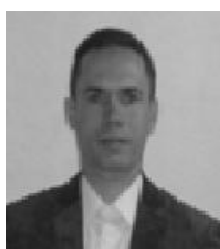

Manuel B. Cuadra Sanz received the Electronic Engineer degree in 1992 from the Universidad of Oriente, Santiago de Cuba, Cuba, and M.Sc degree in Biomedical engineering from the Universidad de Las Villas, Villa Clara, Cuba in 2001. Since 1993 he has been with the Medical Biophysics Center) working on biological signal processing and biomedical instrumentation. His professional research interests are in signal processing, in particular, applied to biomedical applications. 